\title{
Theoretical and Applied Contributions to Robust Stability Analysis of Complex Systems
}

\author{
Baltazar Aguirre-Hernández $\mathbb{D}^{1},{ }^{1}$ Raúl Villafuerte-Segura $\mathbb{D}^{2},{ }^{2}$ Alberto Luviano-Juárez, ${ }^{3}$ \\ and John Cortés-Romero iD ${ }^{4}$ \\ ${ }^{1}$ Departamento de Matemáticas, Universidad Autónoma Metropolitana-Iztapalapa, San Rafael Atlixco 186, CDMX, \\ México 09340, Mexico \\ ${ }^{2}$ Centro de Investigación en Tecnologías de Información y Sistemas, Universidad Autónoma Del Estado de Hidalgo, Pachuca, \\ Hidalgo, Mexico \\ ${ }^{3}$ Instituto Politécnico Nacional, UPIITA, Mexico City, Mexico \\ ${ }^{4}$ Universidad Nacional de Colombia, Bogotá, Colombia
}

Correspondence should be addressed to Baltazar Aguirre-Hernández; bahe@xanum.uam.mx

Received 25 February 2020; Accepted 25 February 2020; Published 10 June 2020

Copyright (C) 2020 Baltazar Aguirre-Hernández et al. This is an open access article distributed under the Creative Commons Attribution License, which permits unrestricted use, distribution, and reproduction in any medium, provided the original work is properly cited.

In the study of the dynamics of a complex system, stability is one of the more interesting aspects since a stability analysis provides the principles and methods useful for engineers, mathematicians, and others to obtain a better understanding of the system. This allows us to understand the nature of the dynamics of the system and propose a new controller or improve control designs, optimizing or increasing performance.

On the other hand, it is common that the mathematical models of dynamic complex systems are, in practice, imprecise due to uncertainties, parametric variations, nonmodeled dynamics, neglected terms in simplifications, etc., among others. Hence, a robust stability analysis for complex systems is essential to achieve results that more closely resemble real-world platforms.

Without a doubt, the robustness of stability is one of the most active research areas in the framework of the analysis and control of dynamic systems.

In the paper "A PI-Type Sliding Mode Controller Design for PMSG-Based Wind Turbine," J. Liu et al. improve the stabilization of a permanent magnet synchronous generator(PMSG-) based wind energy conversion systems (WECS) by means of a PI-Type sliding mode controller. The results are supported by the application of the Lyapunov stability theory and numerical simulations.

The paper "Robust Finite-Time Tracking for Uncertain Linear Systems with Actuator Faults" by X. Peng et al. presents a robust finite-time fault-tolerant control (FTC) scheme for a class of uncertain linear systems in the presence of multiple actuator faults. The adaptive laws were used to compensate the effects of faults and uncertainties. It is shown that the proposed state-feedback model reference adaptive finite-time FTC scheme can guarantee that the tracking error converges to a small neighborhood of the origin in finite time. Simulations are presented for illustrating the proposed design.

In the paper "Research on Optimization of Production Decision Based on Payment Time and Price Coordination," Y. Yan et al. construct a mathematical model of the optimal quote and delivery time of orders between a manufacturer and several retailers, which also includes penalties for late delivery to the manufacturer, in a supply chain. The authors apply the shorter processing time rule (SPT) to solve the problem of production scheduling and use the fact that the gain function (objective function) is a convex function to find the delivery time and the optimal price.

The paper "Ground Attack Strategy of Cooperative UAVs for Multitargets" by Q. Zhang and R. Wei proposes a strategy of a parallel multiview splicing on clouds (PMVSC) unmanned aerial vehicles using precise target recognition and attack and task assignment. Simulations and experiments are performed on an experimental cooperative UAVs platform in order to implement the proposed algorithm. 
In the paper "Risk Analysis of Emergency Based on Fuzzy Evidential Reasoning" by X. Qiao and D. Shi, the proposed analytical reference framework allows the authors to provide an approach based on fuzzy evidential reasoning to analyze, model, and process emergency risks.

The paper "LMI-Based Robust Stabilization of a Class of Input-Constrained Uncertain Nonlinear Systems with Application to a Helicopter Model" by H. Gritli considers the robust stabilization of the pitch dynamics of a helicopter model with uncertainties and disturbances via a theoretical framework based on a LMI approach in order to design a state-feedback control law.

The reachable set bounding for homogeneous nonlinear time-delay systems with bounded disturbance is studied in the paper "Reachable Set Bounding for Homogeneous Nonlinear Systems with Delay and Disturbance" by X. Zhu and Y. Sun. Here, the authors establish a necessary and sufficient condition such that all the system solutions converge asymptotically within a specific ball. Finally, a numerical example is presented to illustrate the proposed theoretical results.

A saturation control with a feedforward term to the trajectory tracking task of a magnetic levitation system connected to a beam mechanism is proposed in the paper "Nested Saturation Function Control of a Magnetic Levitation System" by O. Gutíerrez-Frías et al. The proposal consists in using the flatness of the system to transform the system in an integrator chain, while a controller based on a nested saturated function and a feedforward term are used to force the output trajectories to converge towards the reference trajectory. The closed-loop solution is proven to be locally exponentially stable by means of the second method of Lyapunov. Lastly, numerical simulations prove the effectiveness of the proposal.

The event-triggered strategy for multiple neural networks with parameter uncertainty and time delay is presented in the paper "Global Robust Exponential Synchronization of Multiple Uncertain Neural Networks Subject to Event-Triggered Strategy" by J.-E. Zhang and H. Liu. Here, several sufficient criteria to ensure global robust exponential synchronization of coupling neural networks using matrix inequality techniques are given. Eventually, three numerical examples are offered to illustrate the obtained results.

A fuzzy robust control to mitigate the bullwhip effect in the uncertain closed-loop supply chain with lead times is proposed in the paper "Mitigation of Bullwhip Effect in Closed-Loop Supply Chain Based on Fuzzy Robust Control Approach" by S. Zhang and M. Zhang. For this, the lead times are included in the closed-loop supply chain models, an additional Takagi-Sugeno fuzzy controller for lead times is designed, and a new fuzzy robust control approach is put forward to effectively mitigate the bullwhip effect caused by uncertainties and lead times, and the stability of the closedloop supply chain system using LMIs is ensured.

A trajectory tracking control algorithm based on a fractional-order PD controller for a wheeled mobile robot is presented in the paper "Design, Implementation, and Validation of Robust Fractional-Order PD Controller for
Wheeled Mobile Robot Trajectory Tracking" by L. Zhang et al. For robust fractional-order controller regulation, an improved flat phase property as a robust controller tuning specification is put forward to guarantee the robust flat phase frequency interval width instead of only one flat phase frequency.

The problem of establishing tuning rules to a proportional retarded controller for LTI systems is addressed in the paper " $\sigma$-Stabilization of a Flexible Joint Robotic Arm via Delayed Controllers" by G. Ochoa-Ortega et al. The proposal consists in giving analytic conditions to $\sigma$-stabilize this system class and guarantee a maximal decay rate in the system response. The conditions presented in this paper are tested experimentally in tracking tasks on a underactuated mechanical nonlinear system known as flexible joint robotic arm using a feedback linearization approach.

Concerning the robust stabilization of systems subject to oscillations and possible impacts, a robust LMI-based control approach is proposed in the paper "Robust Position Control of a Two-Sided 1-DoF Impacting Mechanical Oscillator Subject to an External Persistent Disturbance by Means of a State-Feedback Controller," by F. Turkia et al. The authors provide the stabilization conditions of the controlled hybrid dynamics by means of the S-procedure in terms of bilinear matrix inequalities, which are converted into linear matrix inequalities. Numerical simulations for different conditions are provided to show the effectiveness of the proposal.

Considering again fractional-order systems but with an adaptive backstepping control scheme, the paper "Adaptive Neural Network Control of a Class of Fractional Order Uncertain Nonlinear MIMO Systems with Input Constraints" by C. Wang et al. ensures the convergence of tracking errors even with dead-zone and saturation nonlinearities in the controller input. In this work, unknown nonlinear uncertainties are approximated by a radial basis function neural network and the parameters update laws with incommensurate fractional order are used in the controller to compensate those unknown nonlinearities. Two simulation results are presented to validate the efficacy of the proposed scheme.

\section{Conflicts of Interest}

The guest editors declare that they have no conflicts of interest regarding the publication of this Special Issue.

\section{Acknowledgments}

The guest editors would like to thank the authors of all the papers submitted to their interest in this Special Issue and express their enormous thankfulness to the reviewers for their valuable time and effort as well as for providing constructive comments to the authors, making this Special Issue viable.

Baltazar Aguirre-Hernández Raúl Villafuerte-Segura Alberto Luviano-Juárez John Cortés-Romero 